\section{Technology and the single electron}

Marc Kastner

Since their invention, announced in 1948, transistors have become an irreplaceable part of modern life. How can they be made ever tinier? A marriage of lithography and colloidal chemistry is a promising approach.

$\mathrm{T}$ he ability to make progressively smaller structures, developed for the electronics industry, has allowed physicists to study how electrons behave when confined to reduced dimensions. Simultaneously, a new approach is being developed, one in which colloidal chemistry makes it possible to build up nanometre-sized structures atom by atom. The integration of such self-assembled nanostructures with lithographically defined electrodes is likely to reveal new physics, and may also lead to new electronic devices based on quantum-mechanical effects. On page 699 of this issue ${ }^{1}$, Klein et al. report an important step in the merging of these two technologies.

The most exciting discoveries in solidstate physics in the past two decades result from the confinement of electrons. For example, confining electrons to two-dimensional motion in a strong magnetic field creates a new state of matter characterized by the quantum Hall effect. The Hall effect is a voltage that is generated perpendicular to both the current flow and the magnetic field. At low temperature and high field, the ratio of this Hall voltage to the current is accurately quantized. The quantum Hall effect is observed by confining the electrons by man-made material boundaries inside a transistor ${ }^{2,3}$. Similar devices, called field-effect transistors, or FETs, are found in computers and cellular phones.

These FETs are simple devices consisting of three layers - a metal (called the gate), an insulator and a semiconductor (Fig. 1a). There are two contacts attached to the semiconductor, called the source and drain, that allow electrons to enter and exit, and another contact to the gate. When the voltage on the gate $V_{\mathrm{g}}$ is positive, electrons are pulled into the semiconductor, it becomes conducting, and current can flow in response to a voltage applied between source and drain. When $V_{\mathrm{g}}$ is negative, electrons are removed, the semiconductor is an insulator and no current can flow. Thus the FET is a switch that turns on when electrons are added and off when they are removed.

A new kind of transistor is created when electrons are confined to a small volume of space between the source and drain, separated from the two contacts by thin insulating barriers. These barriers allow the electrons to enter or exit only by quantum-mechanical tunnelling. According to quantum mechanics, electrons can penetrate regions of space from which they would classically be excluded. This is called tunnelling. Because of the confinement, both the charge and energy of the electrons become quantized, so the small volume behaves like an artificial atom ${ }^{4,5}$. In general, additional energy is required to add (or subtract) an electron to the confined region; so, for most values of $V_{\mathrm{g}}$, current cannot flow through the transistor. However, for a specific value of $V_{\mathrm{g}}$ the energy of the transistor with $N$ confined electrons is equal to that with $N+1$ confined electrons. For this $V_{\mathrm{g}}$, the charge on the artificial atom can fluctuate and current can flow. This is analogous to the equilibrium between two oxidation states of ions in solution in an electrochemical cell. Because the transistor conducts at one value of $V_{\mathrm{g}}$ for every

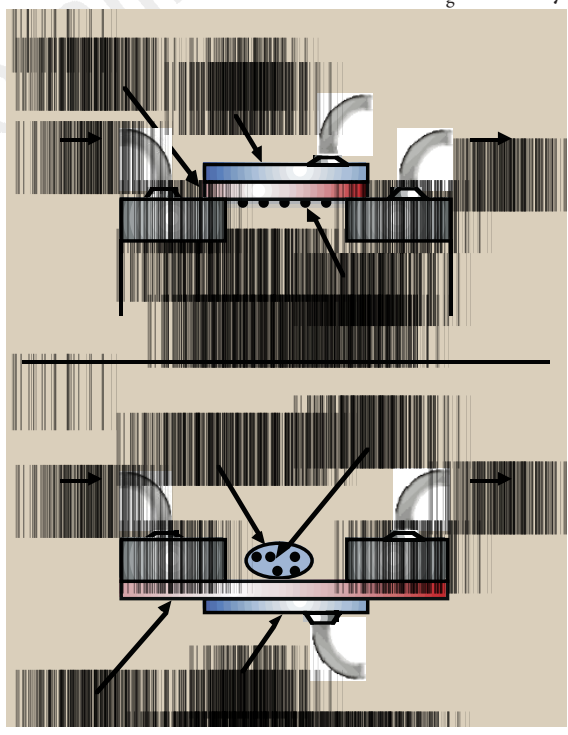

Figure 1 Field-effect and single-electron transistors. a, A field-effect transistor, like that used in computer memories, consists of a semiconductor with metallic source and drain contacts, and a metallic gate electrode nearby, separated from the semiconductor by an insulator. When the voltage on the gate is positive, electrons accumulate in the semiconductor, making it conducting and turning it from the 'off' to the 'on' state. b, A single-electron transistor (SET) is very similar, although the gate is shown on the bottom, as in the device of Klein et al. ${ }^{1}$, discussed here. The semiconductor is a small particle, separated from source and drain by barriers to quantum-mechanical tunnelling. The SET turns on and off again every time an electron is added to the particle. value of $N$, it turns on and off again every time an electron is added to it. That is why it is called a single-electron transistor (SET; Fig. 1b).

SETs have been made in a number of ways using lithographic techniques. ${ }^{4}$ But most of them operate only at very low temperatures, typically less than $10 \mathrm{~K}$. The temperature of operation is proportional to the energy required to add an extra electron to the artificial atom; and, because the latter results primarily from the Coulomb repulsion between electrons, it varies as the inverse radius of the confined region. So, to make SETs that operate at higher temperature, one needs to confine electrons to smaller volumes than are accessible with current lithographic techniques.

In the past few years, however, chemists have learned to make volumes of semiconductor as small as $15 \AA$ in diameter using colloidal chemistry ${ }^{6}$. The slow growth of the nanocrystals allows highly precise size selection, so that huge quantities of nearly identical crystals can be produced. These would make wonderful SETs if one could attach leads to them. But, whereas lithography is limited to creating volumes larger than about $500 \AA$, the nanocrystals are limited to sizes less than about $150 \AA$; if they are larger, they precipitate out of the colloidal suspension. A great technological challenge is to bridge this size discrepancy.

Klein and co-workers ${ }^{1}$ have found a clever way to show that this can indeed be done. They prepare a metal gate with an insulator on top of it, and use lithography to create metal source and drain electrodes on the surface of the insulator. Because only a gap is needed between source and drain, rather than a volume, Klein et al. are able to make the gap as small as about $50 \AA$. When they finally deposit $55-\AA ̊$ semiconductor nanocrystals on the surface, once in a while a single particle falls in the gap, creating a single-electron transistor. This is the first time that a complete SET has been fabricated with a colloidally grown nanocrystal as the confining region. Furthermore, the energy scale observed is quite large.

In itself, the technique is not likely to produce large numbers of useful devices. Nonetheless, Klein and colleagues' successful demonstration-of-principle suggests that the combination of lithographic and chemical techniques might eventually become a practical means for production of the smallest transistors yet.

Marc Kastner is in the Department of Physics,

13-2106, Massachusetts Institute of Technology,

77 Massachusetts Avenue, Cambridge,

Massachusetts 02139-4037, USA.

1. Klein, D. L., Roth, R., Lim, A. K. L., Alivisatos, A. P. \& McEuen, P. L. Nature 389, 699-701 (1997).

2. von Klitzing, K., Dorda, G. \& Pepper, M. Phys. Rev. Lett. 45, 494-497 (1980).

3. Tsui, D. C., Stormer, H. L. \& Gossard, A. C. Phys. Rev. Lett. 48, 1559-1562 (1982).

4. Kastner, M. A. Phys. Today 46, 24-31 (1993).

Ashoori, R. C. Nature 379, 413-419 (1996)

6. Murray, C. B., Norris, D. J. \& Bawendi, M. G. J. Am. Chem. Soc. $115,8706-8715$ (1993) 